\title{
Impact of Metformin on Statin Persistence: a Post Hoc Analysis of a Large Randomized Controlled Trial
}

$\mathrm{J}$ Gen Intern Med 37(1):277-9

DOI: $10.1007 / \mathrm{s} 11606-020-06344-6$

(c) Society of General Internal Medicine 2020

\section{INTRODUCTION}

Optimal cardiovascular disease (CVD) risk reduction is impaired by statin-associated muscle symptoms (SAMS). ${ }^{1-3} \mathrm{Se}-$ vere muscle reactions are well accounted for, yet mild-tomoderate myalgia is common and difficult to track. No valid SAMS therapies exist, and management requires trial-anderror with different statins until one is tolerated. ${ }^{1}$

Metformin may counter-regulate downstream mediators of myalgia through the PI3K/AKT pathway and mitochondrial function. ${ }^{3}$ Our post hoc analysis in a subset of the Action to Control Cardiovascular Risk in Diabetes (ACCORD) trial demonstrated metformin's association with lower odds of myalgia in statin-treated patients. ${ }^{4}$ This post hoc analysis investigates metformin's association with statin nonpersistence in ACCORD-Lipid.

\section{METHODS}

ACCORD-Lipid compared fenofibrate versus placebo in statin-treated patients with type 2 diabetes. ${ }^{5}$ ACCORD-Lipid principally used simvastatin. With simvastatin intolerance, patients were allowed other statins (e.g., atorvastatin). The primary outcome of the present analysis was overall rate of statin persistence between patients taking metformin versus not taking metformin at study-exit. All patients were intended to take statins throughout ACCORD-Lipid. Therefore, patients taking any statin at "study-exit," regardless of symptoms, were considered persistent. Multivariable logistic regression was used to adjust for potential confounders with statin persistence and SAMS susceptibility (Table 1). ${ }^{1,4} \mathrm{~Pa}-$ tients with missing data were excluded from multivariable regression. All comparisons are summarized as OR with 95\% CI. Data were analyzed using SPSS version 24 . The

Prior Presentation The analysis related to the manuscript has been presented at the 2020 American College of Clinical Pharmacy Virtual Annual Meeting; October 19-October 30.

Received September 22, 2020

Accepted November 5, 2020

Published online November 18, 2020
University of South Florida Institutional Review Board determined this study exempt.

\section{RESULTS}

Patient mean age was $\sim 63$ years and $\sim 31 \%$ were women. Fewer study-exit metformin users had baseline history of CVD events, coronary artery bypass graft, or heart failure. ACCORD-Lipid assigned trial arm varied somewhat between study-exit metformin users and non-users. At study-exit, fewer metformin users were also using a dihydropyridine calcium channel blocker. At baseline, more study-exit metformin users were current cigarette smokers.

Statin persistence was significantly higher with metformin (94.4\% versus $89.4 \%)$ compared to without metformin $(n=$ 4773 [86\% of ACCORD-Lipid population], univariate OR: 2.00; 95\% CI: 1.60-2.51). The results remained unchanged when restricting univariate analysis to patients without missing data needed for multivariable regression $(n=3883$ [70\% of ACCORD-Lipid population]; univariate OR: $1.54 ; 95 \% \mathrm{CI}$ : 1.17-2.04). The significantly higher odds of statin persistence with metformin remained unchanged in multivariable regression $(n=3883$; multivariable OR: 1.74 ; 95\% CI: $1.28-2.36$; Table 2).

\section{DISCUSSION}

The present post hoc analysis of ACCORD-Lipid shows metformin use was associated with significantly increased odds of statin persistence. The findings are consistent with metformin's association with lower odds of myalgia. ${ }^{4}$ As a controlled trial using other statins in response to simvastatin intolerance, the overall low statin discontinuation rate was expected. As such, metformin's absolute impact in practice may be larger where patients are not systematically retrialed following SAMS. ${ }^{2}$

As a post hoc analysis, limitations include unknown/ unaccounted for confounders (Table 1) including patients tolerating one medication with common side effects potentially being more likely to tolerate another. Multivariable regression accounted for important confounders such as calcium channel blocker use and time to a macrovascular event, though we were unable to account for exact duration of study participation. Interestingly, lower alanine aminotransferase was associated with statin non-persistence, however, the OR and CI 
Table 1 Potential Confounders in the Present Analysis

\begin{tabular}{llll}
\hline \hline Factors accounted for in multivariable regression & Factors NOT accounted for in multivariable regression \\
\hline Biguanide use* & CVD history & Thyroid function & Pre-existing muscle pathology \\
Age & History of CABG & Vitamin D level & Number of follow-up days in ACCORD-Lipid \\
Gender (female) & History of CVD events & Polypharmacy & Gemfibrozil \\
Race & History of heart failure & Diet & DPP4 inhibitors \\
Body mass index & Drug benefits & Frailty & Macrolides \\
Trial arm & DHP CCB* & Physical activity level & Amiodarone \\
Time to macrovascular event (max 7) & Non-DHP CCB* & Mild-to-moderate myalgia & Immunosuppressants \\
Smoked cigarette (last 30 days) & eGFR* & - & - \\
Current cigarette smoker & ALT* & - & - \\
Smoked > 100 cigarettes in life & Potassium* & - & - \\
Alcohol consumption (amount) & Hemoglobin A1c* & - & - \\
\hline
\end{tabular}

ACCORD, Action to Control Cardiovascular Risk in Diabetes; ALT, alanine aminotransferase; BMI, body mass index; CVD, cardiovascular disease; $C A B G$, coronary artery bypass graft; DHP CCB, dihydropyridine calcium channel blocker; eGFR, estimated glomerular filtration rate

*Variables recorded at baseline unless denoted with, "*”, then exit

†Years to revascularization, myocardial infarction, stroke, hospitalization for heart failure, CVD death, or censor

approached 1.0, thus the association is likely unimpactful. ACCORD-Lipid did not assess mild-to-moderate myalgia in all patients, so we were unable to account for this accordingly. However, we previously demonstrated lower odds of myalgia in a subset of metformin users in ACCORD. ${ }^{4}$ The lower odds of myalgia previously observed $(22-29 \%)^{4}$ aligns with the greater odds of persistence observed. The difference in effect magnitude may be due to the present study's larger sample, assessing patients at study-exit rather than baseline, how clinical trials maintain patients on study therapy, and statin discontinuation potentially relating to issues other than myalgia. Additionally, the current data do not allow for assessing adherence or dose-response relationships.

Metformin remains underused in type 2 diabetes and prediabetes. ${ }^{3,6}$ Validating metformin's impact on SAMS may facilitate statin and metformin use in type 2 diabetes and prediabetes, thus improving CVD risk reduction and diabetes care/prevention. Moreover, metformin may potentially mitigate the increased risk for new-onset diabetes posed by high-intensity statins. ${ }^{1-3}$ Future studies are needed to validate these findings in separate cohorts. For patients without dysglycemia, studies will need to confirm metformin's therapeutic mechanism for SAMS, clarify biomarkers of myalgia versus myopathy, and assess metformin's impact on SAMS via randomized controlled trials.

\section{Acknowledgments:}

The authors would like to thank Randy Rush, PharmD, for assistance in developing the first draft of the manuscript.

Data Source: This manuscript was prepared using ACCORD Research Materials obtained from the NHLBI Biologic Specimen and Data Repository Information Coordinating Center and does not necessarily reflect the opinions or views of the ACCORD researchers or the NHLBI.

Byron Cheon, $\mathrm{MS}^{1}$

Ambuj Kumar, MD, MPH ${ }^{1}$

Athanasios Tsalatsanis, $\mathrm{PhD}^{1}$

Kevin Cowart, PharmD, $M P H^{2}$

Ronald R. Magness, $P h D^{1}$

Srinivas M. Tipparaju, $P h D^{2}$

Nicholas W. Carris, PharmD ${ }^{2}$

${ }^{1}$ Morsani College of Medicine, University of South Florida, 12901 Bruce B Downs Blvd., MDC 30, Tampa, FL 33612, USA

${ }^{2}$ Taneja College of Pharmacy, University of South Florida, 12901 Bruce B Downs Blvd., MDC 30, Tampa, FL 33612, USA

Corresponding Author: Nicholas W. Carris, PharmD; Taneja College of Pharmacy, University of South Florida12901 Bruce B Downs Blvd., MDC 30, Tampa, FL 33612, USA (e-mail: carris@usf.edu).

Table 2 Multivariable Analysis of Metformin's Impact on Statin Persistence $(n=3883)$

\begin{tabular}{|c|c|c|c|c|c|c|c|c|c|}
\hline \multirow{2}{*}{$\frac{\text { Variable }}{\text { Biguanide use* }}$} & \multirow{2}{*}{$\begin{array}{l}\text { OR } \\
1.74\end{array}$} & \multicolumn{3}{|c|}{$95 \% \mathrm{CI}$} & \multirow{2}{*}{$\begin{array}{l}\text { Variable } \\
\text { CVD history }\end{array}$} & \multirow{2}{*}{\begin{tabular}{|l|} 
OR \\
1.00
\end{tabular}} & \multicolumn{3}{|c|}{$95 \% \mathrm{CI}$} \\
\hline & & 1.28 & - & 2.36 & & & 0.05 & - & 18.28 \\
\hline Age & 1.02 & 1.00 & - & 1.04 & History of CABG & 1.28 & 0.70 & - & 2.35 \\
\hline Gender (female) & 1.22 & 0.90 & - & 1.66 & History of CVD events & 1.69 & 0.09 & - & 30.81 \\
\hline Race & 1.01 & 0.89 & - & 1.15 & History of heart failure & 0.60 & 0.33 & - & 1.08 \\
\hline Body mass index & 1.02 & 0.99 & - & 1.05 & Drug benefits & 1.06 & 0.93 & - & 1.22 \\
\hline Trial arm & 1.02 & 0.91 & - & 1.16 & DHP CCB* & 0.80 & 0.53 & - & 1.20 \\
\hline Time to macrovascular event $(\max 7)^{\dagger}$ & 1.02 & 0.93 & - & 1.12 & Non-DHP CCB* & 0.54 & 0.30 & - & 0.99 \\
\hline Smoked cigarette (last 30 days) & 2.98 & 0.41 & - & 21.86 & eGFR* & 1.00 & 1.00 & - & 1.01 \\
\hline Current cigarette smoker & 2.94 & 0.40 & - & 21.83 & ALT* & 1.02 & 1.01 & - & 1.02 \\
\hline Smoked $>100$ cigarettes in life & 0.91 & 0.69 & - & 1.21 & Potassium* & 1.11 & 0.80 & - & 1.55 \\
\hline Alcohol consumption (amount) & 1.04 & 0.99 & - & 1.09 & Hemoglobin A1 c* & 1.03 & 0.92 & - & 1.15 \\
\hline
\end{tabular}

$A L T$, alanine aminotransferase; BMI, body mass index; $C V D$, cardiovascular disease; $C A B G$, coronary artery bypass graft; $D H P C C B$, dihydropyridine calcium channel blocker; eGFR, estimated glomerular filtration rate

*Variables recorded at baseline unless denoted with, "*”, then exit

†Years to revascularization, myocardial infarction, stroke, hospitalization for heart failure, CVD death, or censor 
Funding This study was funded by the William Saunders Endowed Chair, NIH DK119066 to SMT, and NIH HL117341 to RRM. No sponsor played any role in the design or conduct of the study; in the collection, analysis, or interpretation of the data; or in the preparation. review, or approval of the manuscript.

\section{Compliance with Ethical Standards:}

Conflict of Interest: The authors declare that they do not have a conflict of interest.

\section{REFERENCES}

1. Grundy SM, Stone NJ, Bailey AL, et al. 2018 AHA/ACC/AACVPR/ AAPA/ABC/ACPM/ADA/AGS/APhA/ASPC/NLA/PCNA Guideline on the Management of Blood Cholesterol: Executive Summary: A Report of the American College of Cardiology/American Heart Association Task Force on Clinical Practice Guidelines. Circulation. 2019;139:e1046-e1081.
2. Zhang H, Plutzky J, Skentzos S, et al. Discontinuation of statins in routine care settings: a cohort study. Ann Intern Med 2013;158(7):526-34

3. Carris NW, Tipparaju SM, Magness DJ, et al. Pleiotropic effects of metformin to rescue statin-induced muscle injury and insulin resistance: A proposed mechanism and potential clinical implications. Med Hypotheses 2017;107:39-44.

4. Carris NW, Tsalatsanis A, Tipparaju SM, et al. Metformin's impact on statin-associated muscle symptoms: An analysis of ACCORD study data and research materials from the NHLBI Biologic Specimen and Data Repository Information Coordinating Center. Diabetes Obes Metab 2018;20:1994-9.

5. Ginsberg HN, Elam MB, Lovato LC, et al. Effects of combination lipid therapy in type 2 diabetes mellitus. N Engl J Med 2010;362:1563-74.

6. Soric MM, Moorman JM, Boyle JA, Dengler-Crish CM. Prevalence and Predictors of Metformin Prescribing in Adults with Type 2 Diabetes Mellitus: A National Cross-Sectional Study. Pharmacotherapy. 2016;36:715-722.

Publisher's Note: Springer Nature remains neutral with regard to jurisdictional claims in published maps and institutional affiliations. 